\title{
Simulation of Hyperthermic Treatment Using the Matrix of Stripline Applicators
}

\author{
B. Vrbová, L. Víšek
}

\begin{abstract}
This paper describes the design of a microwave stripline applicator for hyperthermic treatment, and the design of an anatomically based biological model, which is a necessary part of hyperthermia treatment planning for measuring the distribution of SAR. In this paper we compare the SAR distribution in a cylindrical homogeneous agar phantom (which has similar characteristics to biological tissue) and in an anatomically based biological model of the femur (which has been developed from a computer tomography scan) using a matrix of two applicators of the same type.
\end{abstract}

Keywords: microwave thermotherapy, SAR, cancer treatment, anatomically based models, hyperthermia applicators.

\section{Introduction}

Thermotherapy is a method based on differences in the behavior of healthy tissue and tumor tissue under enhanced temperatures. One of the most important methods of microwave thermotherapy is hyperthermia. This method works in a temperature interval between 41 and $45^{\circ} \mathrm{C}$ in which cancer cells are destroyed, while healthy cells are able to survive up to $45^{\circ} \mathrm{C}$. If the temperature is increased above $45^{\circ} \mathrm{C}$, coagulation of healthy cells occurs. The self protective mechanism of tumors more than $2 \mathrm{~cm}$ in diameter already fails at a temperature of $41^{\circ} \mathrm{C}$. The blood flow in tumor cells decreases with increasing temperature, and so the temperature in tumor cells increases even more rapidly. Finally, the tumor tissue is destroyed. The duration of a single hyperthermic treatment should not exceed 50 minutes. The level of the hyperthermic dose depends on temperature and time [1].

A hyperthermic set consists of a high power generator, thermal sensors and, especially, the applicator. An electromagnetic wave is generated and the applicator delivers it to the biological tissue, where it is absorbed, because the biological tissue is a lossy dielectric material. Applicators are mainly designed for a working frequency of $434 \mathrm{MHz}$. This is one of the ISM (Industrial, Scientific, Medical) dedicated frequencies [2].

The distribution of the electromagnetic field in the biological tissue can be calculated using a simple, homogeneous model of tissue, which consists of only one type of tissue with defined electric parameters. However, the real tissue is much more complicated. The electromagnetic field diffuses through several types of tissue, so simulations with anatomical models are used for more realistic and more accurate results.

These models can also be used for hyperthermia treatment planning. Treatment planning is a very important procedure in hyperthermia. Before the pa- tient is treated by hyperthermia, several calculations are performed to find out the distribution of the absorbed power in this particular case. The best type of applicator for the treatment is selected according to the location of the tumor. In cases where the tumor is located near to vital organs or other critical areas, calculations with anatomical models of patients are necessary.

\section{Hyperthermia applicator}

The basic parameters of a TEM wave depend mainly on the dielectric parameters of the media through which it propagates (complex permittivity $\varepsilon$ ) and on the working frequency, but not on the cross section dimensions or on the type of transmission line [3].

The applicator discussed here works at a frequency of $434 \mathrm{MHz}$, and is made from a highly conductive material (copper). The sides of the applicator are made from acrylic glass. The TEM wave is transferred along a section of the microwave stripline transmission line with cross-section dimensions of $50 \times 30 \mathrm{~mm}$ and length $80 \mathrm{~mm}$. The horn section of the applicator is $80 \mathrm{~mm}$ in length. The horn aperture is $120 \times 80 \mathrm{~mm}$ (Fig. 1) [4].

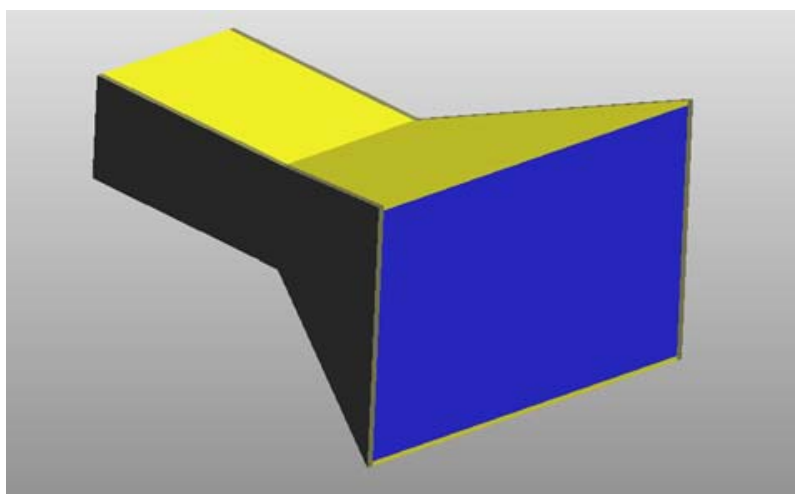

Fig. 1: Model of a stripline applicator 
The length of the applicator is equal to twice the wave length, depending on the relative permittivity of the biological tissue $\left(\varepsilon_{r}=78\right)$. To avoid reflection of the wave back to the generator, the applicator is filled with a suitable dielectric material. In our case, the applicator is filled with distilled water, because $70 \%$ of biological tissue consists of water.

A water bolus is inserted between the applicator and the tissue to improve the transmission of the electromagnetic energy into the tissue. This improves the thermal profile in the biological tissue.

Impedance matching is very important for preventing reflections of electromagnetic energy back to the generator. Reflected energy could destroy the high power output generator. The basic condition for impedance matching is that VSWR has to be lower than 2. The impedance matching of the applicator was simulated using the FDTD simulator (e.g. SEMCAD X EM Field simulator from SPEAG, Schmid \& Partner Engineering AG, Switzerland [6]) in order to find the position and the length of the exciting probe.

The reflection coefficient at working frequency $s_{11}$ is equal to $-32.3 \mathrm{~dB}$, which represents a value of $V S W R=1.05$. The length of the exciting probe is equal to $27 \mathrm{~mm}$ and the distance from the shortcircuited plane is $15 \mathrm{~mm}$.

\section{Anatomically based biological model}

An anatomically based biological model is essential for numerical dosimetry. The numerical model is usually developed from CT scans (Fig. 2).

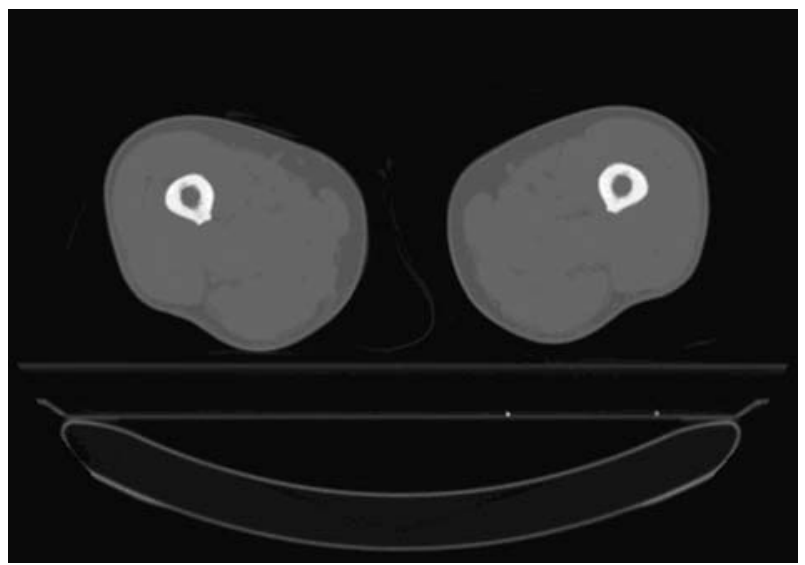

Fig. 2: Computed tomography scan

Dosimetry is used in the design and application of hyperthermia applicators. Dosimetry quantifies the magnitude and distribution of the absorbed electromagnetic energy within biological objects exposed to electromagnetic fields. In RF, the dosimetric quantity, referred to as the specific absorption rate (SAR), is defined as the rate at which energy is absorbed per unit mass. SAR is determined not only by the incident electromagnetic waves but also by the electrical and geometric characteristics of the irradiated subject and nearby objects. It is related to the internal electric field strength and also to the electric conductivity and the density of the tissues. It is therefore a suitable dosimetric parameter, even when a mechanism is determined to be "athermal". SAR distributions are usually determined from calculations on human models.

Rapid progress with computers has enabled highlevel numerical dosimetry to be performed with the aid of high-resolution biological models. The finite difference time domain (FDTD) method is currently the most widely used numerical RF dosimetry method [5].

In order to develop a model for numerical dosimetry, the original gray-scale data must be interpreted into tissue types. This process is known as segmentation. Segmentation of medical images involves partitioning the data into contiguous regions representing individual anatomical objects. This is a prerequisite for further investigations in many computer-assisted medical applications, e.g. individual therapy planning and evaluation, diagnosis, simulation and imageguided surgery.

Segmentation is a difficult task because it is in most cases very hard to separate the object from the image background. This is due to the characteristics of the imaging process as well as the grey-value mappings of the objects themselves. The most common medical image acquisition modalities include CT (computer tomography) and MRI (magnetic resonance imaging) images. MRI or CT provides gray-scale image data in the form of many transverse slices, at a designated spacing, from the head to the feet of the biological body. The resolution in each slice is of the order of several millimeters.

The gray-scale images are first rescaled to produce appropriate voxels. Each voxel in the images then is identified rigorously as belonging to one type of tissue. This is done by assigning to each voxel a red-green-blue code that identifies the discrete tissue type of that particular voxel. This process can be performed with commercial software [7]. All identified transverse images are then combined to obtain a three-dimensional numerical model. Fine adjustment is generally required to connect each slice smoothly in the three orthogonal planes (axial, sagittal and coronal).

DICOM CT scans for a femur model were obtained from Bulovka University Hospital. The femur model has resolution $2 \mathrm{~mm}$, meaning a voxel size of $2 \times 2 \times 2 \mathrm{~mm}$. Each voxel was assigned to one of 3 different tissue types, i.e. muscle, fat and bone (Fig. 3). 


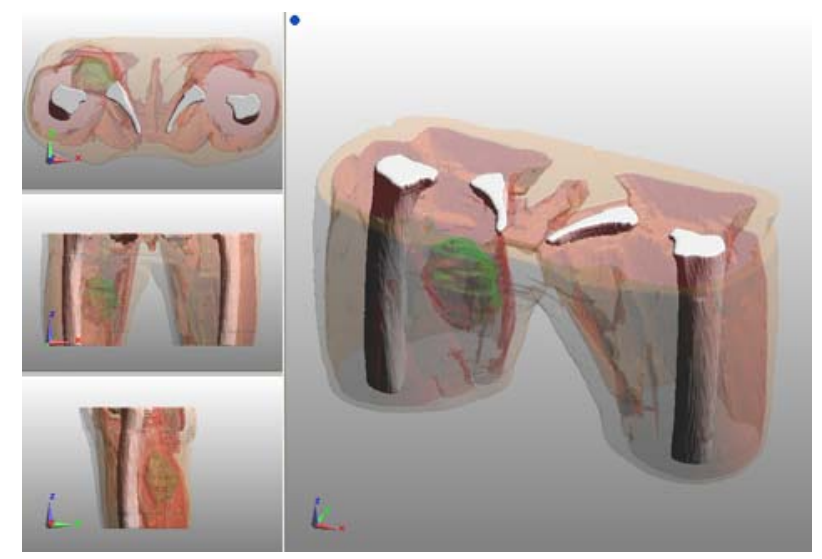

Fig. 3: Anatomical model of a femur

\section{Matrix composition of two applicators}

When the tumours cover a large area of the human body, e.g. on the arm, leg, back or stomach area, we can use applicators of bigger dimensions, or we can create a matrix of several smaller applicators.

In our case, we chose to use a matrix. We chose the matrix composition of two applicators of the same type. In the first case we put two applicators on a cylindrical agar phantom next to each other (Fig. 4).

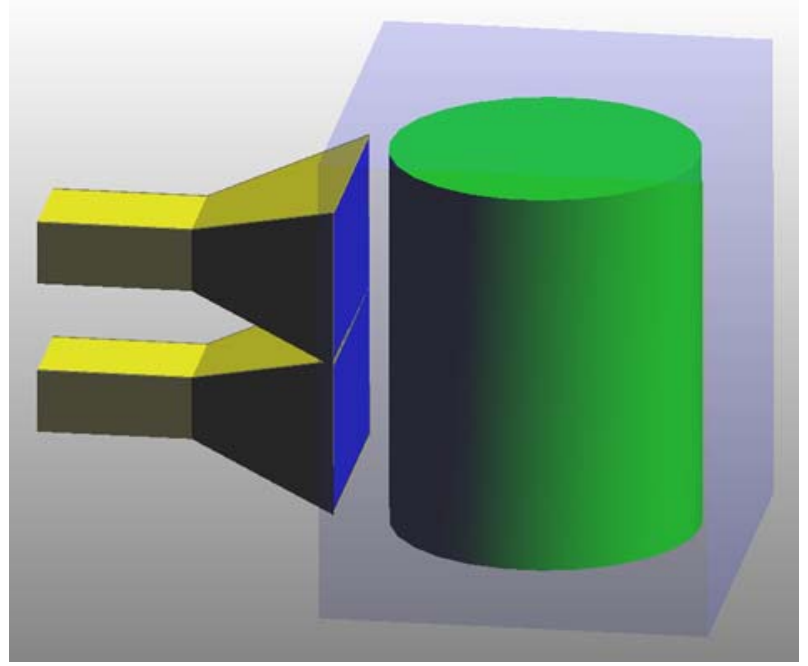

Fig. 4: Matrix of two applicators on a cylindrical agar phantom

This homogeneous agar phantom represents a femur from a human body, and its dielectric properties are shown in the following table (Tab. 1). The radius of the cylindrical agar phantom is $8 \mathrm{~cm}$.

In the second case, we placed a matrix of two applicators on an anatomically-based biological model (Fig. 5), the dielectric parameters of which are listed in Table 1.
Table 1: Dielectric properties at a frequency of $434 \mathrm{MHz}$ [8]

\begin{tabular}{|l|c|c|}
\hline Name & Conductivity $[\mathrm{S} / \mathrm{m}]$ & $\begin{array}{c}\text { Relative } \\
\text { permittivity }\end{array}$ \\
\hline Agar & 0.80 & 54.00 \\
\hline Cortical Bone & 0.09 & 13.07 \\
\hline Muscle & 0.80 & 56.86 \\
\hline Fat & 0.04 & 5.56 \\
\hline
\end{tabular}

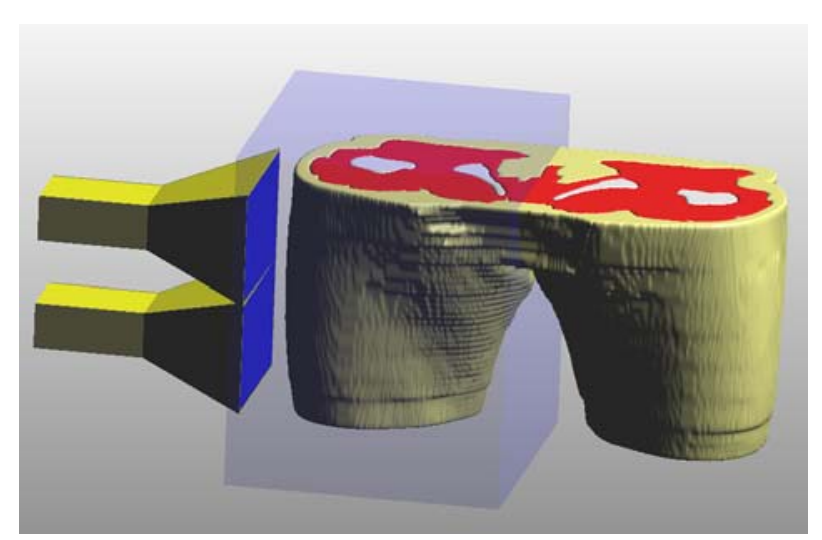

Fig. 5: Matrix composition of two applicators on an anatomically-based biological model

\section{Results}

The results of the simulations of SAR distribution are shown in the following pictures. The maximum SAR distribution is situated in the middle of the two applicators in the homogeneous agar phantom (Fig. 6).

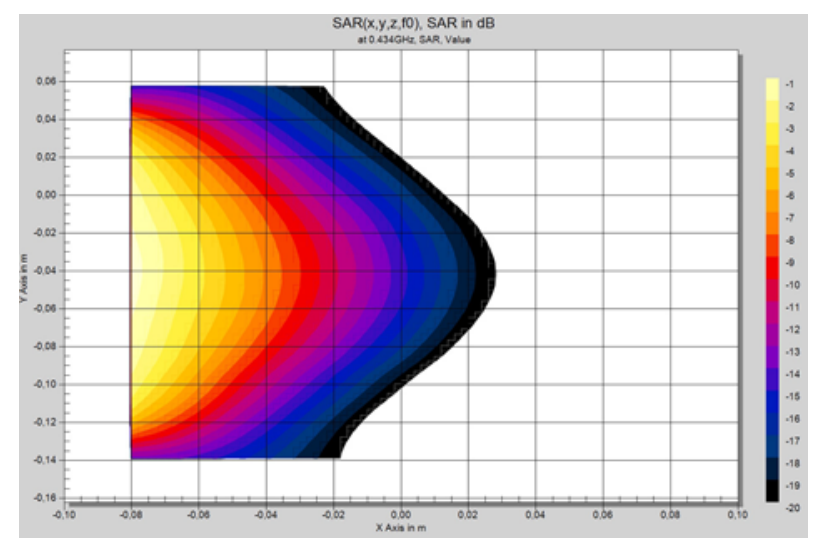

Fig. 6: SAR distribution in the agar phantom in the longitudinal cutting plane

A comparison of the SAR distribution in the agar phantom and in the anatomic model shows that the SAR distributions acquire their maximum at the same locations, but the shape of the SAR distribution in the anatomical phantom is influenced by the fat and bone (Fig. 7). 


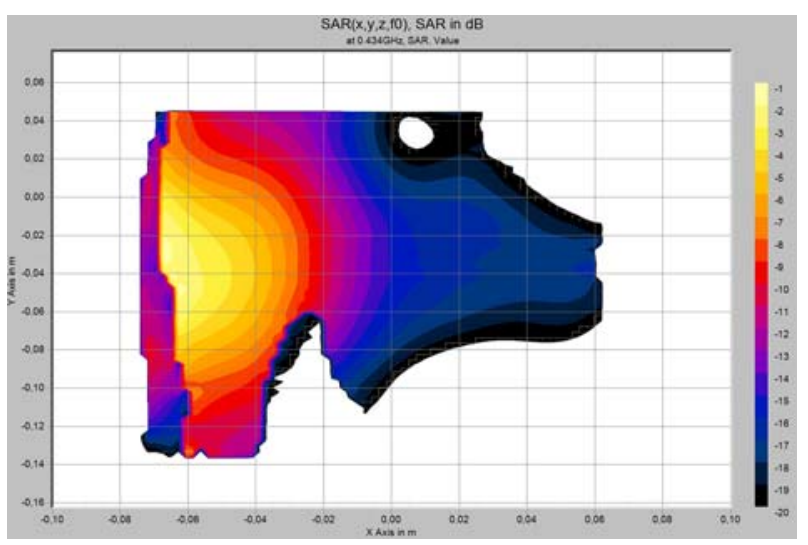

Fig. 7: SAR distribution in the anatomical model in the longitudinal cutting plane

Fat has a lower permittivity value than muscle, and therefore a small part of the energy is absorbed in the fat and most of the energy goes into another layer, into the muscle. Muscle behaves as a lossy environment, so the energy is absorbed there. Bones also affect the shape of the SAR distribution, as can be seen in the transversal section of the femur (Fig. 8).

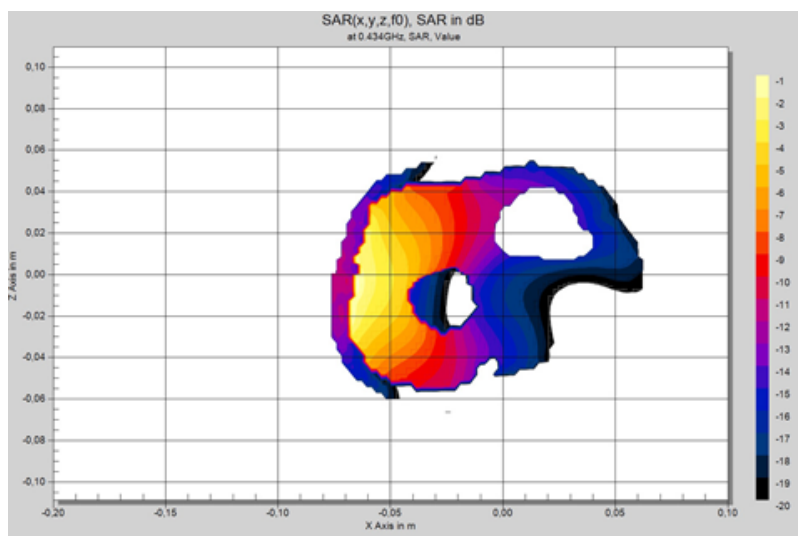

Fig. 8: SAR distribution in the transversal cutting plane of the anatomic model

Bones, like fat, have a low permittivity value (they contain a small amount of water), but the SAR value is almost equal to zero in this place, because bone behaves as a lossless environment and most of the energy goes through the bones.

Fig. 9 shows the shape of the SAR distribution in the transversal layer of the homogeneous agar phantom.

Simulations of SAR distribution shows that microwave stripline applicators can be used to treat tumors located under the surface of tissue. Simulation of the SAR distribution in an agar phantom is used for testing applicators. However, in cases of hyperthermic treatment planning where the tumor is located near to vital organs or other critical areas, calculations must be made with an anatomical model of the patient.

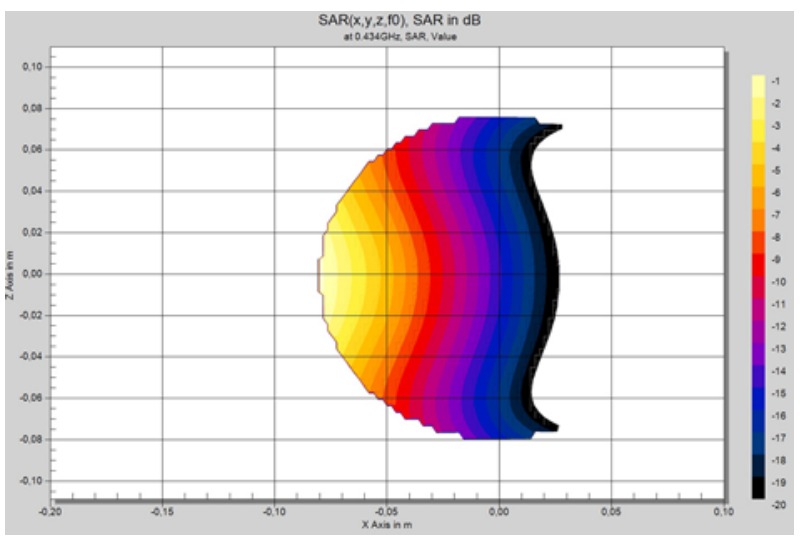

Fig. 9: SAR distribution in the transversal cutting plane of an agar phantom

\section{Conclusions}

A set of several applicators can be used in clinical practice to treat tumors covering a large area of the human body. In future the treatment of tumors can be improved by setting the amplitude and the phase of the applicators.

\section{Acknowledgement}

This research has been supported by the Grant Agency of the Czech Republic project: "Non-standard application of physical fields - analogy, modelling, verification and simulation" (102/08/H081).

\section{References}

[1] Falk, H. M., Issels, R. D.: Hyperthermia in Oncology. International Journal of Hyperthermia, Vol. 17, No. 1, 1-18, 2001.

[2] Vrba, J.: Medical Applications of Microwaves. Prague (Czech Republic), 2007.

ISBN 978-80-01-02705-9.

[3] Vrba, J.: Introduction to Microwave Technology. Prague (Czech Republic), 2007.

ISBN 978-80-01-03670-9.

[4] Vrbová, B.: Diploma thesis. Microwave stripline applicator for local thermotherapy. Prague, 2009.

[5] Fujiwara, O., Wang, J.: Electromagnetics in Biology, Springer Japan, chapter on Radiofrequency Dosimetry and Exposure Systems, 2006, p. $223-225$.

[6] Schmid \& Partner Engineering AG, [Online], URL: http://www.semcad.com/, 2009.

[7] 3D-DOCTOR, 3D Imaging, Modelling, Rendering and Measurement Software, [Online], URL: http://www.ablesw.com/3d-doctor, 2009. 
[8] Gabriel, C.: Compilation of the Dielectric Properties of Body Tissues at RF and Microwave Frequencies, Brooks Air Force Technical Report, AL/OETR-1996-0037.

\section{About the authors}

Barbora VRBOVÁ was born in Nové Zámky, Slovakia, on March 28, 1984. She received her MSc degree in biomedical engineering from the Czech Technical University in Prague in 2009. She deals with radiometric methods for verifying the biological effects of EM fields.

Lukáš VÍŠEK was born in Vysoké Mýto in 1982 and received his MSc degree from the Czech Technical Uni- versity in Prague in February 2006. He is currently a postgraduate student at the Department of Electromagnetic Field at the Faculty of Electrical Engineering. His present work is on developing an exposure system for unrestrained small animals which will serve for research on the non-thermal effects of electromagnetic fields and hyperthermic applicators.

Barbora Vrbová

Lukáš Víšek

E-mail: vrbovbar@fel.cvut.cz,

visekluk@fel.cvut.cz

Dept. of Electromagnetic Field

Faculty of Electrical Engineering

Czech Technical University

Technická 2, 16627 Praha, Czech Republic 